\title{
Missing Energy B Decays at the Belle II Experiment
}

\author{
Mario Merola* on behalf of the Belle II Collaboration \\ Università di Napoli Federico II and INFN Napoli, Italy \\ E-mail: mario.merola@na.infn.it
}

The Belle II experiment at the SuperKEKB collider is a major upgrade of the KEK "B factory" facility in Tsukuba, Japan. The machine is designed to produce an instantaneous luminosity of $8 \times 10^{35} \mathrm{~cm}^{-2} \mathrm{~s}^{-1}$, and the experiment is expected to accumulate a data sample of about $50 \mathrm{ab}^{-1}$. With this amount of data, decays sensitive to physics beyond the Standard Model can be studied with unprecedented precision. One promising set of modes are physics processes with missing energy due to a neutrino in the final state, such as the semileptonic and leptonic $B$ meson decays $B \rightarrow X_{u} \ell v, B \rightarrow D^{(\star)} \tau \nu$, and $B \rightarrow \ell v(\gamma)$. In addition, the rare decay $B \rightarrow K^{(*)} v \bar{v}$ provides one of the cleanest experimental probes of the favour-changing neutral current process $b \rightarrow s v \bar{v}$. This report discusses the expected sensitivities of the Belle II data sample to these decays.

The International Conference on B-Physics at Frontier Machines - BEAUTY2018

6-11 May, 2018

La Biodola, Elba Island, Italy

${ }^{*}$ Speaker. 


\section{Introduction}

In the past years, the Belle and BABAR experiments, operating at the electron-positron colliders KEKB (Tsukuba, Japan) and PEP-II (Stanford, US) respectively, have offered important insights into the flavour sector of particle physics. The achievements of the two collaborations, which collected together an integrated luminosity of about $1.5 \mathrm{ab}^{-1}$, led to the 2008 Nobel prize for physics being awarded to Kobayashi and Maskawa for their theory of $C P$ violation within the Standard Model (SM). In order to answer some of the open questions in the flavour physics sector, particularly to probe the nature of the tensions with the SM predictions in the current observations, additional data is needed. For this purpose the KEKB collider has been upgraded to reach an instantaneous luminosity of $8 \times 10^{35} \mathrm{~cm}^{-2} \mathrm{~s}^{-1}$, such that a sample of $50 \mathrm{ab}^{-1}$ can be accumulated. Furthermore, the Belle detector has undergone a major upgrade, to cope with the significantly higher level of beam-induced backgrounds, while maintaining or improving the performance.

In the SuperKEKB collider, beams of electrons and positrons with energy of $7 \mathrm{GeV}$ and $4 \mathrm{GeV}$, respectively, will mainly collide at a centre-of-mass energy of $10.58 \mathrm{GeV}$, which corresponds to the peak of the $\Upsilon(4 S)$ resonance. Data taking at other $\Upsilon(n S)$ resonances is also scheduled. At the design luminosity, the machine will produce $10^{10} \mathrm{~B}$ pairs per year, therefore, becoming the most prolific $B$-factory ever built. The gain of a factor of 40 in instantaneous luminosity foreseen by the SuperKEKB collider with respect to its predecessor KEKB is obtained by means of higher beam currents $(\sim 2 \times)$ and strong beam squeezing at the interaction point $(\sim 20 \times)$. To achieve this big improvement and at the same time keep the beam instabilities under control, the machine has faced several upgrades involving the radio frequency cavities, the dipole and quadrupole magnets, the beam pipe, and the interaction point (IR) optics, which is referred to as the nano-beam scheme [1].

The Belle detector underwent important upgrades: the Vertex Detector (VXD) added two layers of pixel silicon detector and increased its polar angle coverage, the Central Drift Chamber (CDC) has smaller cell sizes, there are two new particle identification devices (PID) in the barrel (Time-of-Propagation counter) and in the endcaps (Aerogel Ring Imaging Cherenkov detector), the installation of faster electronics for the Electromagnetic Calorimeter (ECL), and the replacement of the resistive plate chambers in the endcap of the muon and $K_{L}$ detectors (KLM) with scintillators instrumented with silicon photomultipliers. The mechanical structure and the superconducting solenoid providing a magnetic field of $1.5 \mathrm{~T}$ are left unchanged. These features lead to several improvements of the detector performances, from the vertex resolution and efficiency of $K_{S}$ and slow pions reconstruction (VXD, CDC), to the pion/kaon separation (PID) and machine background rejection (ECL). Furthermore, the improved hermeticity of the Belle II detector allows more precise measurements of the missing four-momentum in $B$ decays that have one or more neutrino in the final state, as will be detailed in the next section.

The first collisions have been recorded on April $26^{\text {th }} 2018$ with the Phase-2 Belle II detector, which is without the VXD, and the data taking continued until the mid July. Afterwards, the almost complete VXD ${ }^{1}$ will be installed and from February 2019 Belle II will start taking data with this detector configuration (Phase-3).

\footnotetext{
${ }^{1}$ Only one layer of pixel detectors will be ready for installation before the start of Phase- 3 .
} 


\section{Reconstruction of missing energy $B$ decays at Belle II}

The Belle II experiment offers unique capabilities to study the $B$ decays with neutrinos in the final state, with respect to experiments at hadron colliders. The center-of-mass energy of the collisions is precisely known, within a few $\mathrm{MeV}$, and running at the $\Upsilon(4 S)$ resonance peak produces a very clean sample of $B$ meson pairs, with relatively low tracks multiplicity and detector occupancy. In addition, the Belle II detector is capable of high reconstruction efficiencies for both charged and neutral particles. These features allow the full reconstruction of one of the two $B$ mesons (called $\left.B_{\text {tag }}\right)$, in order to infer the four-momentum of the other $B$ meson $\left(B_{\text {sig }}\right)$. An advanced technique Belle II uses to reconstruct the $B_{\text {tag }}$, via hadronic or semileptonic $B$ decays, is the Full Event Interpretation (FEI). It is an extension of the Full Reconstruction (FR) used in Belle [2] and makes use of thousands of exclusive decay modes to train several multivariate classifiers in a hierarchical approach, starting from identifying final-state particles as leptons, charged pions, kaons and photons, to the intermediate particles, such as $D_{(s)}^{(*)}$ and $J / \psi$ mesons, $K_{S}^{0}$ and $\pi^{0}$, ending with the reconstruction of the $B$ meson candidate. It has been shown, using the Belle II GEANT4-based [3] simulation and considering the hadronic tagging modes, that the FEI has improved upon the Belle FR performance by increasing the tagging efficiency from $0.2 \%$ to $0.5 \%$, at a fixed $B_{\text {tag }}$ purity of $25 \%$ [4].

The continuum background events $e^{+} e^{-} \rightarrow q \bar{q}, \tau^{+} \tau^{-}$contaminate the $B \bar{B}$ pairs data sample, but are usually strongly suppressed by exploiting their different topology (particles back-to-back) with respect to the isotropic distribution of particles from $B \bar{B}$ events. The so called "continuum suppression" makes use of this topological difference by using the CLEO cones and Kakuno-SuperFox-Wolfram moments [5, 6], and is employed in most studies of $B$ meson decay.

It is worth introducing here the observable $E_{\mathrm{ECL}}$ (or $E_{\mathrm{Extra}}$ ), on which almost all of the missing energy $B$ decay studies rely upon. It is defined as the residual energy deposited in the ECL once we have subtracted all the clusters associated with the $B_{\text {tag }}$ and $B_{\text {sig. }}$. It is expected to peak at $0 \mathrm{GeV}$ for correctly reconstructed $\Upsilon(4 S)$ decays, while it shows a broader distribution, peaking at larger values, for mis-reconstructed signal events or for background events. It has been verified with the Belle II full simulation that the impact of the photon background coming from the beams and the interaction region (synchrotron radiation, radiative Bhabha, two-photons processes) on $E_{\mathrm{ECL}}$ can be controlled by exploiting the distinctive features of the background calorimetric clusters compared to the energy deposits coming from the $\Upsilon(4 S)$.

\section{Semileptonic $B$ decays and $\left|V_{u b}\right|$ determination}

The $\left|V_{u b}\right|$ determinations from inclusive $\left(B \rightarrow X_{u} l \bar{v}\right)$ and exclusive $(B \rightarrow \pi \ell v$ for example) measurements exhibit a clear tension (Fig. 1, left). The inclusive studies of $B \rightarrow X_{u} l \bar{v}$, where $X_{u}$ is any hadronic final state coming from the fragmentation of the $u$ quark, are typically based on measurements of partial branching fractions in various fiducial kinematic regions. The $\left|V_{u b}\right|$ determinations show a slight dependency on the fiducial region which may be due to differences in theory treatment or to experimental mismodelling of the signal and background components, of which the most important is $B \rightarrow X_{c} l \bar{v}$. 
Belle II has performed a full simulation study of one of the most promising exclusive decay modes, $B \rightarrow \pi \ell v$ [4]. Two different strategies have been explored for this study, using the hadronic tagging of the $B$ meson with FEI or considering an inclusive tagging (the so called untagged method). In the last case instead of explicitly reconstructing the companion $B$, firstly the signal $B$ candidates are reconstructed with kinematical and angular requirements on pions and leptons, and then a selection is applied on clusters and tracks not used for the signal reconstruction (the Rest Of Event). The details of the analysis are omitted in this report (as well for the next sections), at the end the signal yield measurement relies on the reconstructed missing mass squared $m_{m i s s}^{2}=m_{v}^{2}$, which is expected to peak at $0 \mathrm{GeV}^{2} / \mathrm{c}^{4}$. The extrapolated uncertainties on $\left|V_{u b}\right|$ in the tagged and untagged analyses, with the increasing Belle II collected dataset, are depicted in Fig. 1 (right). The $\left|V_{u b}\right|$ extraction from the branching ratio $(\mathscr{B})$ measurement strongly relies on the $B$ decays form factors calculations in Lattice QCD (LQCD) [7]: in Fig. 1 (right) the current LQCD estimation refers to the world average reported by the Flavour Lattice Averaging Group (FLAG) [8], the 5 years projection assumes a factor of 2 reduction of the lattice QCD uncertainty on the semileptonic form factors, while the 10 years projection assumes a factor of 5 reduction. In addition, these estimates assume that the electromagnetic corrections contribute a $1 \%$ uncertainty, which is added in quadrature to the QCD uncertainty.
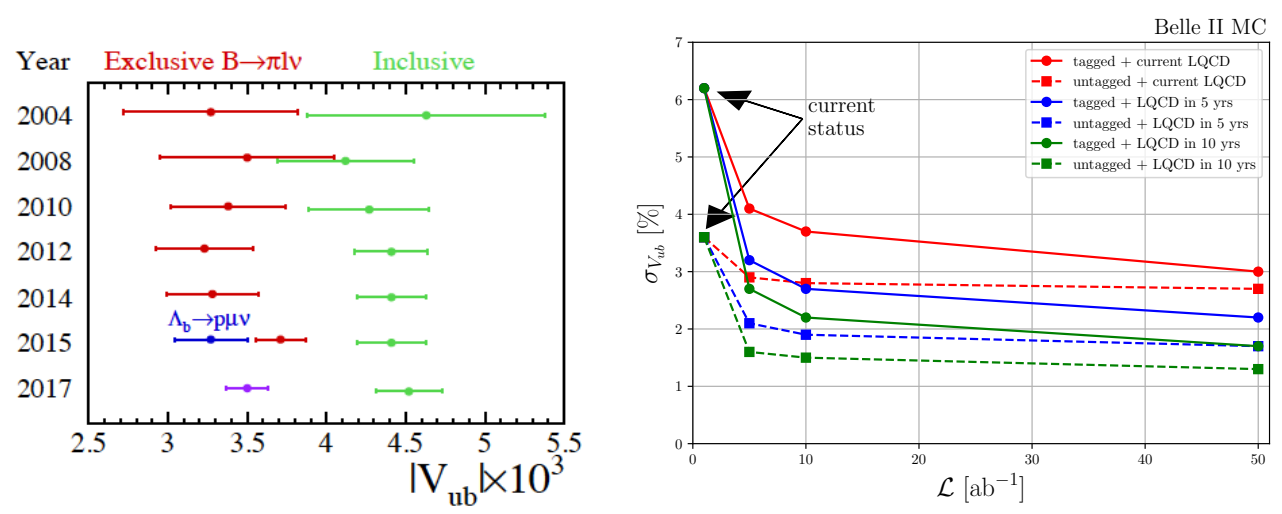

Figure 1: $\left|V_{u b}\right|$ determinations from exclusive and inclusive semileptonic $B$ meson decays (left). Projections of $\left|V_{u b}\right|$ uncertainty to various luminosity values including LQCD uncertainty expectations for $B \rightarrow \pi \ell v$ tagged and untagged modes (right).

\section{Semileptonic $B$ decays into charmed mesons and $\mathbf{R}\left(\mathbf{D}^{(*)}\right)$}

The semileptonic $B$ decays $B \rightarrow D^{(*)} \ell v$ are a clear test of the Standard Model, as New Physics (NP) can largely affect both the $\mathscr{B}$ and the $\tau$ polarization, through diagrams involving a charged Higgs boson [9] or leptoquarks [10]. The observables $R_{D^{(*)}}=\mathscr{B}\left(B \rightarrow D^{(*)} \tau \nu\right) / \mathscr{B}\left(B \rightarrow D^{(*)} \ell v\right)$, where $\ell=\mu, e$, are usually considered, as the ratio reduces the experimental uncertainties on the tagging efficiencies, the theoretical uncertainties on the semileptonic form factors and the uncertainty from the $\left|V_{c b}\right|$. The current world average of the two ratios [11], combining measurements performed by Belle, BABAR and LHCb, is $R_{D}=0.397 \pm 0.040$ (stat.) \pm 0.028 (syst.) and $R_{D^{*}}=0.310 \pm 0.015$ (stat.) \pm 0.008 (syst.), which are significantly above the SM expectations [12] 


\begin{tabular}{ccc}
\hline & $5 \mathrm{ab}^{-1}$ & $50 \mathrm{ab}^{-1}$ \\
\hline$R_{D}$ & $( \pm 6.0 \pm 3.9) \%$ & $( \pm 2.0 \pm 2.5) \%$ \\
$R_{D^{*}}$ & $( \pm 3.0 \pm 2.5) \%$ & $( \pm 1.0 \pm 2.0) \%$ \\
$P_{\tau}\left(D^{*}\right)$ & $\pm 0.18 \pm 0.08$ & $\pm 0.06 \pm 0.04$ \\
\hline
\end{tabular}

Table 1: Expected precision on $R_{D^{(*)}}$ and $P_{\tau}\left(D^{*}\right)$ at Belle II, given as the relative uncertainty for $R_{D^{(*)}}$ and absolute on $P_{\tau}\left(D^{*}\right)$. The two values refer to the statistical and systematic uncertainties, respectively.

of $R_{D}=0.299 \pm 0.003$ and $R_{D^{*}}=0.257 \pm 0.003$, corresponding to a deviation of approximately $4 \sigma$. The Belle measurements using hadronic or semileptonic tag are based on $E_{\mathrm{ECL}}$ for the signal extraction, and on the squared missing mass $M_{\text {miss }}^{2}$ and the angle between the reconstructed signal $B$ and the $D^{*} \ell$ system, $\cos \theta_{B-D^{*} \ell}$, to separate the signal mode $B \rightarrow D^{(*)} \tau \nu$ from the normalization mode $B \rightarrow D^{(*)} \ell v$. One of the most important systematic uncertainties affecting the measurements originates from the knowledge of the $B \rightarrow D^{* *} \ell v$ background. For precision measurements at Belle II, dedicated measurements of $B \rightarrow D^{* *} \ell v$ with a large data sample are essential.

The $\tau$ polarization is defined as $P_{\tau}\left(D^{(*)}\right)=\left(\Gamma^{+}-\Gamma^{-}\right) /\left(\Gamma^{+}+\Gamma^{-}\right)$, where $\Gamma^{+(-)}$is the decay rate with the $\tau$ helicity $+1 / 2(-1 / 2)$. The most recent Belle measurement [13] is in agreement with the SM to within $2 \sigma$.

Table 1 summarizes the estimates of the precision of $R_{D^{(*)}}$ and $P_{\tau}\left(D^{(*)}\right)$ with 5 and $50 \mathrm{ab}^{-1}$ collected at Belle II, based on existing results from Belle and the expected experimental improvements with the upgraded detector [4]. In Fig. 2 the expected precisions with $50 \mathrm{ab}^{-1}$ at Belle II are compared with the current measurements and with the SM predictions. It is worth emphasizing that with the first 5 to $10 \mathrm{ab}^{-1}$ collected by Belle II, which should be within about 2 years from the start of the phase- 3 , we will be able to potentially observe a $5 \sigma$ discrepancy from the SM expectations. NP scenarios will also be tested studying differential branching fraction distributions, for example as a function of the momentum transfer to the lepton pair, $q^{2}=\left(p_{\tau}+p_{v}\right)^{2}=\left(p_{B}-p_{D^{(*)}}\right)^{2}$.
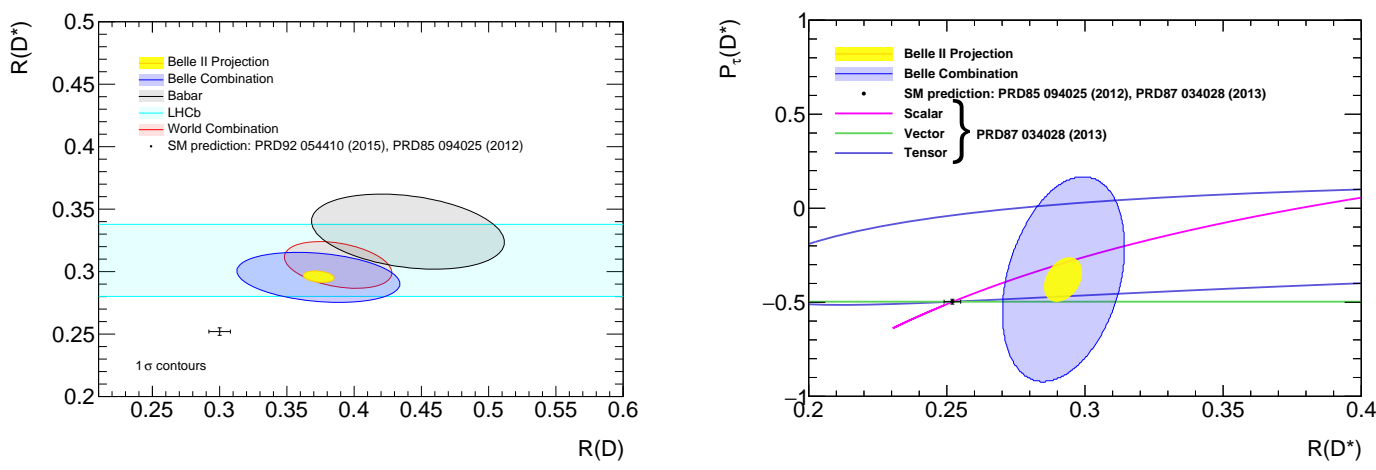

Figure 2: Expected Belle II constraints on the $R_{D} v s . R_{D^{*}}$ plane (left) and the $R_{D^{*}} v s . P_{\tau}\left(D^{*}\right)$ plane (right) compared to existing experimental constraints. The SM predictions are indicated by the black points with theoretical error bars.

\section{Leptonic $B$ decays}

The branching fractions of $B^{-} \rightarrow \ell^{-} \bar{v}_{\ell}$ are hierarchical in the lepton masses due to the he- 


\begin{tabular}{clccc}
\hline & Integrated Luminosity $\left(\mathrm{ab}^{-1}\right)$ & 1 & 5 & 50 \\
\hline \multirow{3}{*}{ hadronic tag } & statistical uncertainty $(\%)$ & 29 & 13 & 4 \\
& systematic uncertainty $(\%)$ & 13 & 7 & 5 \\
& total uncertainty (\%) & 32 & 15 & 6 \\
\hline \multirow{3}{*}{ semileptonic tag } & statistical uncertainty (\%) & 19 & 8 & 3 \\
& systematic uncertainty (\%) & 18 & 9 & 5 \\
& total uncertainty (\%) & 26 & 12 & 5 \\
\hline
\end{tabular}

Table 2: Expected uncertainties on the $B^{-} \rightarrow \tau^{-} \bar{v}_{\tau}$ branching fraction for different luminosity scenarios with hadronic and semileptonic tag methods.

licity suppression of the $B$ meson decay, in the absence of new physics. The predicted values in the $\mathrm{SM}[14,11]$ are $\mathscr{B}\left(B^{-} \rightarrow \tau^{-} \bar{v}_{\tau}\right)=(7.7 \pm 0.6) \times 10^{-5}, \mathscr{B}\left(B^{-} \rightarrow \mu^{-} \bar{v}_{\mu}\right)=(3.5 \pm 0.3) \times 10^{-7}$, $\mathscr{B}\left(B^{-} \rightarrow e^{-} \bar{v}_{e}\right)=(8.1 \pm 0.6) \times 10^{-12}$. NP contributions, as predicted by the Higgs doublet models [15], can increase or decrease these values depending on the interference with the SM processes.

\section{$5.1 B \rightarrow \tau \nu$}

The tauonic mode has been measured by Belle and BABAR, and the last Belle measurement [16], combining the results obtained in the hadronic and semileptonic tag, reached a precision of $24 \%$ on the $\mathscr{B}$. The Belle II experiment has carried out a study of measurement prospects with the full simulation [4], reconstructing the $B_{\text {tag }}$ in hadronic modes with the FEI, and reconstructing the tau lepton in four single-prong decay modes, specifically $\mu^{-} v_{\tau} \bar{v}_{\mu}, e^{-} v_{\tau} \bar{v}_{e}, \pi^{-} v_{\tau}, \pi^{-} \pi^{0} v_{\tau}$. The most important part of the event selection is the reduction of the beam background photons component exploiting the properties of the calorimeter clusters originated from physics photons: they are more energetic, in time with the bunch crossing, and usually are associated with few calorimeter crystals, while beam-induced photon showers exhibit a larger spread of energy deposits. The signal is extracted with a maximum likelihood fit to the $E_{\mathrm{ECL}}$ distribution (Fig. 3, left). A toy MC study has shown that with $1 \mathrm{ab}^{-1}$ of data Belle II will be able to measure $\mathscr{B}\left(B^{-} \rightarrow \tau^{-} \bar{v}_{\tau}\right)$ with a relative precision of $29 \%$, which corresponds to a significance of $3.4 \sigma$ when only considering the statistical uncertainty. The measurement projections with 5 and $50 \mathrm{ab}^{-1}$, including the systematic uncertainties and extrapolating from the Belle measurement for the semileptonic tag, are summarized in Table 2. The main expected contributions to the systematic uncertainties come from the knowledge of the background models, the $\mathscr{B}$ of the backgrounds peaking in $E_{\mathrm{ECL}}$ and the tagging efficiencies. Nevertheless most of these uncertainties will be strongly reduced with increasing luminosity as they can be determined from data control samples.

A dedicated study has been performed to evaluate the impact of the beam background on the measurement, and in particular on the $E_{\mathrm{ECL}}$ resolution. The Belle II MC with superimposed nominal beam background has been compared to the Belle $\mathrm{MC}$ used for the measurement with hadronic tag [17], see Fig. 3. The comparison shows that the impact of the increased beam background is almost negligible, with a slightly better resolution at Belle II, thanks to the upgraded detector and the advanced decay reconstruction techniques used in the new experiment.

In conclusion, the full simulation study indicates that Belle II will be able to observe the 


\begin{tabular}{ccc}
\hline Integrated luminosity & $5 \mathrm{ab}^{-1}$ & $50 \mathrm{ab}^{-1}$ \\
\hline Absolute uncertainty $\left(\times 10^{-6}\right)$ & $+0.56-0.53$ & $+0.18-0.17$ \\
\hline
\end{tabular}

Table 3: Expected absolute statistical uncertainty in $10^{-6}$ at Belle II for a simulated branching fraction of $B^{-} \rightarrow \ell^{-} \bar{v}_{\ell} \gamma(\ell=\mu, e)=5.0 \times 10^{-6}$.

$B^{-} \rightarrow \tau^{-} \bar{v}_{\tau}$ decay, using the hadronic tag, with a significance above the $5 \sigma$ level after collecting about $3 \mathrm{ab}^{-1}$ of data.
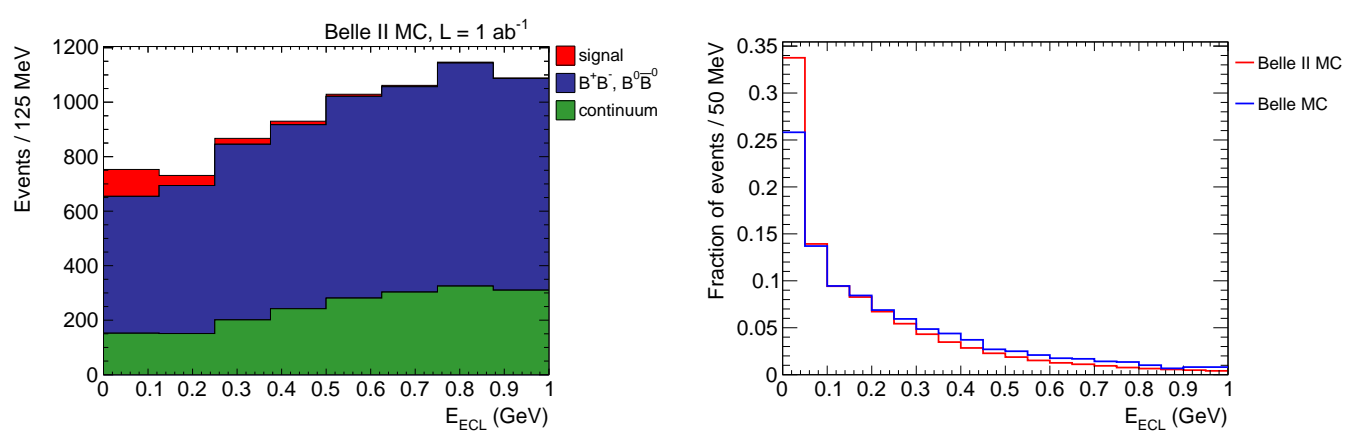

Figure 3: Left: $E_{\mathrm{ECL}}$ distribution for signal (red), $B \bar{B}$ background (blue) and continuum (green). The events are normalised to an integrated luminosity of $1 \mathrm{ab}^{-1}$. Right: Comparison of signal $E_{\mathrm{ECL}}$ distribution for Belle II (red) and the Belle measurement with hadronic tag (blue).

\section{$5.2 B \rightarrow \mu v$ and radiative $B \rightarrow \ell v \gamma$}

Despite its $\mathscr{O}\left(10^{-7}\right)$ branching fraction, the $B^{-} \rightarrow \mu^{-} \bar{v}_{\mu}$ decay is very promising at Belle II, given its distinct topology, where the muon is monochromatic in the signal $B$ rest frame. This feature has been exploited by the Belle searches $[18,19]$ using both tagged and untagged techniques, to separate the signal from other $B$ decays. The projections based on the Belle measurement show that Belle II should reach the $5 \sigma$ observation of this channel with approximately $6 \mathrm{ab}^{-1}$ of data.

The radiative decay lifts the helicity suppression of the purely leptonic $B$ decays up to a $\mathscr{B} \sim$ $10^{-5}$. These decays allow the measurement of a very important input to QCD factorization schemes for non-perturbative calculation of non-leptonic $B$ meson decays: the emission of the photon probes the first inverse moment $\lambda_{B}$ of the light-cone distribution amplitude of the $B$ meson [20]. A recent study performed on Belle MC has shown that the Belle II FEI tagging algorithm can increase the signal yield by a factor of three, with the same dataset size, compared to predictions based on the Belle analyses. In Table 3 the projections of the statistical uncertainties on the $\mathscr{B}\left(B^{-} \rightarrow \ell^{-} \bar{v}_{\ell} \gamma\right)(\ell=$ $\mu, e)$ expected at Belle II are shown.

\section{Search for $B \rightarrow K^{(*)} v \bar{v}$}

The decays $B \rightarrow K^{(*)} v \bar{v}$ are prohibited at tree level in the SM, such that they can only occur through a flavour-changing neutral current box or penguin diagram. NP models [21, 22, 23, 24] might contribute to the loops or at the tree level, increasing the SM branching fraction of $10^{-6}$ to 


\begin{tabular}{lcc}
\hline Observables & $5 \mathrm{ab}^{-1}$ & $50 \mathrm{ab}^{-1}$ \\
\hline $\mathscr{B}\left(B^{+} \rightarrow K^{+} v \bar{v}\right)$ & $30 \%$ & $11 \%$ \\
$\mathscr{B}\left(B^{0} \rightarrow K^{* 0} v \bar{v}\right)$ & $26 \%$ & $9.6 \%$ \\
$\mathscr{B}\left(B^{+} \rightarrow K^{*+} v \bar{v}\right)$ & $25 \%$ & $9.3 \%$ \\
\hline
\end{tabular}

Table 4: Expected sensitivities to the $K^{(*)} v \bar{v}$ modes with different Belle II datasets.

$10^{-5}$ by a factor of 50 . Experimental searches have been carried out by both Belle and BABAR Collaborations $[25,26]$ and no evidence for signal has been found. Fig. 4 summarizes the current upper limits and the SM expectations.

Belle II has performed a full simulation study [4] reconstructing the $B_{\text {tag }}$ meson with FEI in hadronic decay modes, and selecting the signal channel $K^{*+} \rightarrow K^{+} \pi^{0}$. After applying selection criteria on kinematics and event-shape variables, in particular exploiting the missing energy and the missing momentum in the event, the signal efficiency and background yields have been estimated in the signal region of $E_{\mathrm{ECL}}$. The beam background impact on the measurement has been evaluated as well, comparing the Belle II simulation superimposed to nominal background with a background-free configuration: a negligible effect has been observed both in terms of the $E_{\mathrm{ECL}}$ variable distribution and signal significance.

Projections with 5 and $50 \mathrm{ab}^{-1}$ collected at Belle II, with both hadronic and semileptonic tags, are reported in Table 4 . With about $18 \mathrm{ab}^{-1}$, Belle II will be able to reach the $5 \sigma$ observation of the $B \rightarrow K^{(*)} v \bar{v}$ channels, and with the full dataset the fraction of longitudinally polarized $K^{*}$, which is helpful in distinguishing different NP scenarios, may also be measured with a precision of $20 \%$.

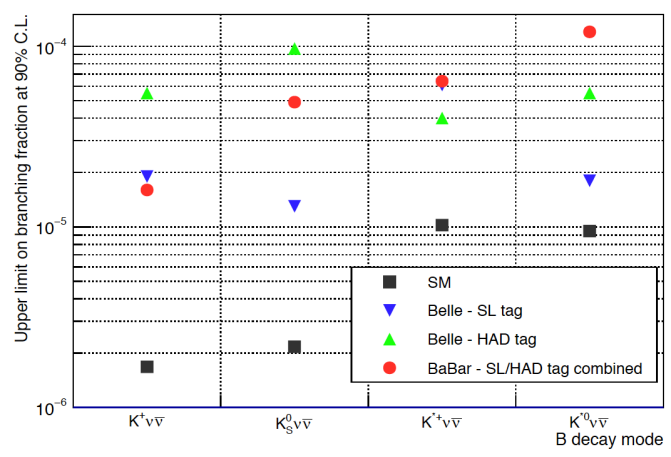

Figure 4: Experimental upper limits and expected SM branching fraction for the decays $B \rightarrow K_{(S)}^{(*)} v \bar{v}$.

\section{Conclusions}

The performed major upgrades of the $e^{+} e^{-}$KEKB collider and the Belle detector will allow the collection of $10^{10} B \bar{B}$ pairs per year at the peak instantaneous luminosity of $8 \times 10^{35} \mathrm{~cm}^{-2} \mathrm{~s}^{-1}$. In addition, Belle II can efficiently identify and reconstruct the charged and neutral particles produced in the collisions. Furthermore, the advanced reconstruction techniques being developed by Belle II will allow the measurement of missing energy decays of $B$ mesons with an unprecedented 
precision. Within the first two years of data taking, corresponding to an integrated luminosity of 5 to $10 \mathrm{ab}^{-1}$, Belle II will possibly address the lepton-flavour-universality violation by precisely measuring $R_{D^{(*)}}$, as well as the tension between measurements of $\left|V_{u b}\right|$ made using samples of inclusive and exclusive semileptonic $B$ decays $B \rightarrow X_{u} l \bar{v}$. It will also have a strong discovery potential for rare processes suppressed in the SM, such as $B \rightarrow \tau \nu, B \rightarrow \mu \nu, B \rightarrow \ell v \gamma$, and the flavour-changing neutral current decays $B \rightarrow K^{(*)} v \bar{v}$. A detailed discussion of the Belle II detector and machine background measurements, software tools, and physics program will be published in 2018 in the "Belle II physics book".

\section{References}

[1] M. Bona et al. (SuperB Collaboration), SuperB: A High-Luminosity Asymmetric $e^{+} e^{-}$Super Flavor Factory. Conceptual Design Report (2007), 0709.0451 [hep-ex] .

[2] M. Feindt, F. Keller, M. Kreps, T. Kuhr, S. Neubauer, D. Zander, A. Zupanc, A Hierarchical NeuroBayes-based Algorithm for Full Reconstruction of B Mesons at B Factories, NIM A 654 (2011) $432[1102$. 3876 [hep-ex] ].

[3] J. Allison et al. (Geant4 Collaboration), Geant4 developments and applications, IEEE Trans. Nucl. Sci. 53 (2006) 270;

S. Agostinelli et al. (Geant4 Collaboration), Geant4: A simulation toolkit, Nucl. Instrum. Meth. A 506 (2003) 250 .

[4] E. Kou, P. Urquijo (Belle II Collaboration and B2TiP theory community), The Belle II Physics Book, to be submitted to Prog. Theor. Exp. Phys..

[5] D. M. Asner et al. (CLEO Collaboration), Search for Exclusive Charmless Hadronic B Decays, Phys. Rev. D 53 (1996) 1039 [hep-ex/9508004].

[6] A.J. Bevan, B. Golob, T. Mannel et al., The Physics of the B Factories, Eur. Phys. J. C 74 (2014) 3026 [1406.6311[hep-ex]].

[7] J. M. Flynn, T. Izubuchi, T. Kawanai, C. Lehner, A. Soni, R. S. Van de Water, and O. Witzel, $B \rightarrow \pi \ell v$ and $B_{s} \rightarrow K \ell v$ form factors and $\left|V_{u b}\right|$ from 2+1-flavor lattice $Q C D$ with domain-wall light quarks and relativistic heavy quarks, Phys. Rev. D 91 (2015) 074510 [1501. 05373 [hep-lat] ].

[8] S. Aoki et al., Review of lattice results concerning low-energy particle physics, Eur. Phys. J. C 77(2) (2017) 112 [1607.00299 [hep-lat]].

[9] M. Tanaka, Charged Higgs Effects on Exclusive Semi-tauonic B Decays, Z. Phys. C 67 (1995) 321 [hep-ph/9411405].

[10] Y. Sakaki, R. Watanabe, M. Tanaka, and A. Tayduganov, Testing leptoquark models in $\bar{B} \rightarrow D^{(*)} \tau \bar{v}$, Phys. Rev. D 88 (2013) 094012 [1309. 0301 [hep-ph] ].

[11] Y. Amhis et al. (Heavy Flavor Averaging Group), Averages of b-hadron, c-hadron, and $\tau$-lepton properties as of summer 2016, Eur. Phys. J. C 77(12) (2017) 895 [1612.07233 [hep-ex] ]. Updated results and plots available at https://hflav.web.cern.ch

[12] F. U. Bernlochner, Z. Ligeti, M. Papucci, and D. J. Robinson, Combined analysis of semileptonic $B$ decays to $D$ and $D^{*}: R\left(D^{(*)}\right),\left|V_{c b}\right|$, and new physics, Phys. Rev. D 95 (2017) 115008, [Erratum: Phys. Rev. D 97 (2018) 059902] [1703. 05330 [hep-ph] ]. 
[13] S. Hirose et al. (Belle Collaboration), Measurement of the $\tau$ Lepton Polarization and $R\left(D^{*}\right)$ in the Decay $\bar{B} \rightarrow D^{*} \tau^{-} \bar{v}_{\tau}$, Phys. Rev. Lett. 118 (2017) 211801 [1612. 00529 [hep-ex] ].

[14] R. J. Dowdall, C. T. H. Davies, R. R. Horgan, C. J. Monahan, and J. Shigemitsu, B-meson decay constants from improved lattice NRQCD and physical $u, d$, s and c sea quarks, HPQCD, Phys. Rev. Lett. 110(22) (2013) 222003 [1302.2644 [hep-lat]].

[15] A. Crivellin, C. Greub, A. Kokulu, Explaining $B \rightarrow D \tau \nu, B \rightarrow D^{*} \tau \nu$ and $B \rightarrow \tau \nu$ in a two Higgs doublet model of type III, Phys. Rev. D 86 (2012) 054014 [1206. 2634 [ hep-ph ] ].

[16] B. Kronenbitter et al. (Belle Collaboration), Measurement of the branching fraction of $B^{+} \rightarrow \tau^{+} \nu_{\tau}$ decays with the semileptonic tagging method, Phys. Rev. D 92 (2015) 051102(R) [1503.05613 [hep-ex]].

[17] I. Adachi et al. (Belle Collaboration), Evidence for $B^{-} \rightarrow \tau^{-} \bar{v}_{\tau}$ with a Hadronic Tagging Method Using the Full Data Sample of Belle, Phys. Rev. Lett. 110(13) (2013) 131801 [1208.4678 [hep-ex]].

[18] Y. Yook et al. (Belle Collaboration), Search for $B^{+} \rightarrow e^{+} v_{e}$ and $B^{+} \rightarrow \mu^{+} v_{\mu}$ decays using hadronic tagging, Phys. Rev. D 91 (2015) 052016 [1406.6356 [hep-ex] ].

[19] A. Sibidanov et al. (Belle Collaboration), Search for $B^{-} \rightarrow \mu^{-} \bar{v}_{\mu}$ Decays at the Belle Experiment, 1712.04123 [hep-ex] .

[20] M. Beneke, G. Buchalla, M. Neubert, and Christopher T. Sachrajda, QCD factorization for exclusive, non-leptonic B meson decays: General arguments and the case of heavy-light final states, Nucl. Phys. B 591 (2000) 313 [hep-ph/ 0006124 ].

[21] W. Altmannshofer, A. J. Buras, D. M. Straub, and M. Wick, New strategies for New Physics search in $B \rightarrow K^{*} v \bar{v}, B \rightarrow K v \bar{v}$ and $B \rightarrow X(s) v \bar{v}$ decays, JHEP 0904 (2009) 022 [0 902.0160 [hep-ph ] ].

[22] G. Buchalla, G. Hiller, and G. Isidori, Phenomenology of nonstandard Z couplings in exclusive semileptonic $b \rightarrow$ s transitions, Phys. Rev. D 63 (2000) 014015 [hep-ph / 0006136 ].

[23] X. -Q. Li, Y. -D. Yang, and X. -B. Yuan, Anomalous tqZ coupling effects in rare B-and K-meson decays, JHEP 1203 (2012) 018 [1112.2674 [hep-ph ] ].

[24] J. H. Jeon, C. S. Kim, J. Lee, and C. Yu, Exclusive $B \rightarrow M v \bar{v}\left(M=\pi, K, \rho, K^{*}\right)$ decays and leptophobic Z' model, Phys. Lett. B 636 (2006) 270 [hep-ph/ 0602156$].$

[25] J. Grygier et al. (Belle Collaboration), Search for $B \rightarrow h v \bar{v}$ decays with semileptonic tagging at Belle, Phys. Rev. D 96(9) (2017) 091101 [1702.03224 [hep-ex] ].

[26] J. P. Lees et al. (BaBar Collaboration), Search for $B \rightarrow K^{(*)} v \bar{v}$ and invisible quarkonium decays, Phys. Rev. D 87(11) (2013) 112005 [1303. 7465 [hep-ex] ]. 